\title{
Benefits of Screening Mammography
}

\author{
Wendie A. Berg, MD, PhD \\ American Radiology Services, Johns Hopkins Green Spring, Lutherville, MD 21093; phone \\ 410-583-2703; fax 410-583-2797 \\ Wendie A. Berg: wendieberg@gmail.com
}

Breast cancer is a leading cause of premature death for women in their forties, and mammographic screening significantly reduces the risk of death due to breast cancer. The latter was the conclusion of the United States Preventive Services Task Force (USPSTF) meta-analysis of the randomized controlled trials (RCTs) of screening mammography ${ }^{1}$. The USPSTF concluded, however, that the absolute benefit from routine mammographic screening of women in their forties was too low to offset the harms. As a woman in her forties, and a radiologist specializing in breast imaging, I find their conclusions puzzling.

RCTs underestimate the true benefits of mammographic screening ${ }^{2}$. RCTs assess mortality reduction among women randomized to the group invited to be screened rather than those who actually participated in screening. In an RCT, an invited woman who never has a mammogram and dies of breast cancer is counted as having been screened, just as a woman in the control group who decides to have a mammogram outside the trial is counted as having not been screened. In several RCTs, mammographic quality was quite poor or only a single mammographic view was obtained, both of which reduce cancer detection.

Two observational studies among women who underwent screening mammography (with the current standard of two views of each breast), comprising millions of person-years of observation of both screened and unscreened populations, reported a 30 to $40 \%$ reduction in breast cancer mortality for women in their forties ${ }^{3,4}$. However, these high-quality observational data were not considered by the USPSTF, which limited its focus to RCTs. For women aged 40 to 49 in a screening program, on average, about 1 in 460 will be diagnosed with breast cancer in any given year; during that decade of life, one in 69 women will be diagnosed with invasive breast cancer ${ }^{2}$. The incidence of breast cancer increases with age, particularly above age 40, with the rates at ages 45 to 49 virtually identical to those at ages 50 to 54 , and no abrupt change at age $50^{2}$. At least a third of potential years of life lost to breast cancer occur among women who died of breast cancer after being diagnosed in their forties ${ }^{5}$. Over 40,000 women die from breast cancer each year, of whom $18 \%$ were diagnosed during their forties (personal communication, Carol DeSantis, MPH, American Cancer Society, December 10, 2009); assuming a 30\% reduction in breast cancer deaths due to screening, annual mammography prevents over 2000 deaths per year among women diagnosed in their forties in the United States.

Mammographic screening also increases the chance of detecting breast cancer small enough to be treated by lumpectomy instead of mastectomy and decreases the need for chemotherapy. Harms of treating more advanced disease and the increased risk of recurrence for women not screened by mammography were not considered by the USPSTF.

Disclosure

WAB is a consultant to Naviscan, Inc. and an uncompensated member of the medical advisory board of Medipattern, Inc. 


\section{Potential harms of mammographic screening}

There are downsides associated with screening, but most women would not consider these harms. In a survey of 479 women, $63 \%$ felt that 500 or more false positives per life saved was a reasonable tradeoff ${ }^{6}$. According to estimates from the Breast Cancer Surveillance Consortium, for every 1,000 women in her forties screened once with mammography in the United States, 80 to 100 will be called back for additional evaluation, usually consisting of additional mammographic views and often ultrasound; 45 to 65 of those recalled have nothing of concern ("false positives"); 20 may be asked to return in six months, with the area followed having less than a $2 \%$ chance of malignancy; 15 will be recommended for biopsy, usually a needle biopsy; and 2 to 5 of women biopsied will be found to have breast cancer ${ }^{2}$. There is a minimal theoretical concern that " 0.05 " women could develop cancer 10 to 30 years later from the small amount of radiation exposure from the mammogram; such "risk" is even lower for older women exposed to mammography?

A few indolent cancers that would not spread during the lifetime of the woman may be detected on the first mammogram, and can result in potentially unnecessary treatment. Such overdiagnosis is likely uncommon (estimated at $4 \%$ of ductal carcinoma in situ) among new or enlarging findings on subsequent annual screens as the lesion has shown itself to be growing. ${ }^{2}$

\section{Screening only women in their forties who are at increased risk}

Seventy-five percent of breast cancer is diagnosed in women with no obvious risk factors for breast cancer; therefore, the USPSTF recommendation against routine screening for women in their forties in the absence of risk factors is problematic. Research is ongoing to develop and test other screening modalities for use in addition to mammography in women at increased risk ${ }^{8}$. In women with dense breasts, in whom mammographic sensitivity is reduced, addition of screening ultrasound provides a $28 \%$ absolute increase in detection of node-negative breast cancer ${ }^{8}$. In women at high risk, screening MRI improves detection of early breast cancer by $56 \%$ beyond mammography and ultrasound combined ${ }^{8}$. Such increases in sensitivity come with significant increases in recall rates and needle biopsies. On average, $11 \%$ of needle biopsies prompted only by screening ultrasound and $40 \%$ of biopsies prompted only by screening MRI prove malignant ${ }^{8}$.

\section{Clinical breast examination}

Clinical breast examination is much less effective than ultrasound or MRI, increasing early breast cancer detection by only $3 \%$ beyond mammography, with only $1 \%$ of suspicious findings proving malignant ${ }^{9}$. Going forward, resources might be better spent on ultrasound in women with dense breasts than on clinical breast examination, although currently there is a shortage of trained personnel in breast ultrasound. The USPSTF recommended against teaching breast self examination, due to an increase in false positive findings and lack of demonstrated benefit. However, women who notice changes in their breast(s) should bring that information to their clinician's attention.

\section{Annual or Biennial Screening}

Breast cancer in younger women tends to be more biologically aggressive. Estimates are that preclinical breast cancer has a $16 \%$ chance of spreading to lymph nodes within one year in a woman in her forties vs $7 \%$ for a woman in her fifties and $5 \%$ for a woman in her sixties ${ }^{10}$. To achieve optimal benefit from mammography, a woman in her forties would need to be screened every year. 


\section{Continue screening mammography after age 74 ?}

As with women aged 40 to 74 , screening mammography decreases the stage of disease at diagnosis and deaths due to breast cancer among women in their seventies and eighties ${ }^{8}$. Average life expectancy of a woman in her eighties is 8.6 years, so screening can be continued as long as a woman is in reasonably good health and would pursue treatment if cancer is identified.

In summary, screening mammography is the only test to date proven to reduce deaths due to breast cancer. Annual mammographic screening is appropriate starting at age 40, provided the woman is willing to accept the downsides of false positives, including being recalled for more imaging and the possibility of a needle biopsy for a finding that is not breast cancer. The overwhelming majority of women are willing to accept these downsides as part of the process of saving lives otherwise lost to breast cancer ${ }^{6}$.

\section{Acknowledgments}

Special thanks to Robert A. Smith, PhD, Cancer Control Sciences Department, American Cancer Society, Atlanta, GA; R. Edward Hendrick, PhD, Department of Radiology, University of Colorado, Denver, CO, and Daniel B. Kopans, MD, Department of Radiology, Massachusetts General Hospital, Harvard University School of Medicine, for helpful discussions.

WAB is supported by grants from The Avon Foundation and National Cancer Institute, U01 CA079778 and U01 CA89008.

\section{References}

1. Nelson HD, Tyne K, Naik A, Bougatsos C, Chan BK, Humphrey L. Screening for Breast Cancer: An Update for the U.S. Preventive Services Task Force. Ann Intern Med. Nov 17; 2009 151(10): 727-737. [PubMed: 19920273]

2. [accessed December 11, 2009] http://sbi-online.org/associations/8199/files/ Detailed_Response_to_USPSTF_Guidelines-12-11-09-Berg.pdf

3. Coldman A, Phillips N, Warren L, Kan L. Breast cancer mortality after screening mammography in British Columbia women. Int J Cancer. Mar 1; 2007 120(5):1076-1080. [PubMed: 17149701]

4. Tabar L, Yen MF, Vitak B, Chen HH, Smith RA, Duffy SW. Mammography service screening and mortality in breast cancer patients: 20-year follow-up before and after introduction of screening. Lancet. Apr 26; 2003 361(9367):1405-1410. [PubMed: 12727392]

5. Shapiro, S.; Venet, W.; Strax, P.; Venet, L. The Health Insurance Plan Project and its Sequelae. Baltimore: Johns Hopkins Press; 1988. Periodic Screening for Breast Cancer.

6. Schwartz LM, Woloshin S, Sox HC, Fischhoff B, Welch HG. US women's attitudes to false positive mammography results and detection of ductal carcinoma in situ: cross sectional survey. BMJ. Jun 17; 2000 320(7250):1635-1640. [PubMed: 10856064]

7. Committee to Assess Health Risks from Exposure to Low Levels of Ionizing Radiation, National Research Council of the National Academies. Health risks from exposure to low levels of ionizing radiation - BEIR VII, Phase 2. Washington, DC: National Academies Press; 2006.

8. Berg WA. Tailored supplemental screening for breast cancer: what now and what next? AJR Am J Roentgenol. Feb; 2009 192(2):390-399. [PubMed: 19155400]

9. Bancej C, Decker K, Chiarelli A, Harrison M, Turner D, Brisson J. Contribution of clinical breast examination to mammography screening in the early detection of breast cancer. J Med Screen. 2003; 10(1):16-21. [PubMed: 12790311]

10. Swedish Cancer Society and the Swedish National Board of Health and Welfare. Breast-cancer screening with mammography in women aged 40-49 years. Int J Cancer. Dec 11; 1996 68(6):693699. [PubMed: 8980168] 